\title{
Environmental justification of the distribution of pressure drop in a dead-end low-pressure gas network
}

\author{
Anna Malysheva ${ }^{1, *}$ \\ ${ }^{1}$ Moscow State University of Civil Engineering, 26, Yaroslavskoe ch., 129337, Moscow, Russia
}

\begin{abstract}
The gas supply system is determined by the classes of the elements of the gas transmission network associated with the pressure of the pumped natural gas. Laying gas pipelines in urban areas requires sufficient space around the pipes as a safety zone. The gas pipelines of the first level include gas communications in which the methane pressure is high or medium. To eliminate dead-end sections, gas pipelines are backed up (duplicating individual segments or ringing). The creation of a dead-end network is allowed only in small settlements. Dead-end network is a gas pipeline branching in various directions to gas consumers. Each section of the branched network has a one-way power supply.
\end{abstract}

\section{Introduction}

In branched low-pressure gas networks, gas is supplied to the consumption node in one direction; therefore they are dead-end networks [1-3]. Thus, consumers connected to branched networks have a one-way power supply. If an element of a branched network is out of work, then all consumers that are connected behind this element will not receive gas. In a dead-end network, transit costs are unambiguously distributed across sections [4]. A change in the diameter of a section of a branched network does not affect the distribution of costs in other sections and only leads to a change in pressure at the initial point of the network.

Hydraulic losses and the diameter of the sections depend on the length of the network. So, the hydraulic losses of pipelines along the length are reduced as a result of its minimization, the diameters of the sections can be reduced as well and, as a consequence, the metal consumption of the system decreases [5]. Since transit costs in the network are uniquely determined, therefore, the estimated costs for all sections are known. Thus, each section is characterized by two unknowns: the diameter di and the pressure loss.

\section{Methods}

If the number of sections of the dead-end network is $P$, then the total number of unknowns will be $2 P$. For each section, one can write the equation of hydraulic losses:

\footnotetext{
*Corresponding author: mani495@mail.ru
} 


$$
\Delta p_{i}=a \frac{Q_{i}^{\alpha}}{d_{i}^{\beta}} l_{i},
$$

where $\Delta p_{i}$ is pressure loss at the section;

$a$ is a gas property dependent coefficient;

$l_{i}$ is a section length;

$d_{i}$ is a section diameter;

$\alpha, \beta$ are system indicators, which generally depend on the gas flow mode and pipe roughness.

Such equations can be $p$, therefore, the remaining number of unknowns will be $p$.

Distribution gas networks rely on a constant design pressure drop [6]. With this principle in mind, additional equations can be written in a following way:

$$
\sum_{1}^{k} \Delta p_{i}-\Delta p=0 .
$$

These equations establish that in each direction from the feed point to the end point " $k$ " the sum of the pressure losses should be equal. The number of such equations is equal to the number of endpoints " $k$ ".

The remaining number of unknowns (extra unknowns) is $f=p-k$, since there is only one input in each node of the branched network, therefore, the number of nodes, excluding the first, is equal to the number of sections, that is:

$p=m-1$.

Using the ratio, one can write:

$f=p-k=m-1-k=m-(1+k)$.

The equation that determines the number of extra unknowns has the following interpretation: the total number of nodes $m$, the number with a given $(1+k)$, that is, the first and all end nodes. Thus, the number of extra unknowns is equal to the number of nodes with unsettled pressures.

\section{Results}

To determine unnecessary unknowns, an additional condition must be specified. This condition is the minimization of the reduced cost functions [7-9]. Taking into account the fact that operating costs with a slight change in diameters practically remain unchanged, it is possible to minimize the cost of gas pipelines or even the metal consumption.

Analyzing the process of minimizing the cost function from the standpoint of the distribution of losses over the sections of gas pipelines, it can be argued that the pressure losses between successively located sections should be distributed so that the total cost of the network is minimal [10]. This suggests that the optimal solution will correspond to the optimal shape of the piezometer, which should be determined while minimizing the cost function.

\begin{tabular}{|c|c|c|c|}
\hline & I & 2 & II \\
\cline { 2 - 3 } & $\mathrm{Q}=720$ & $\mathrm{Q}=120$ & $\mathrm{Q}=60$
\end{tabular}

Fig. 1. Building cold supply system classification. 
Table 1. Preliminary calculation of the gas pipeline diameter.

\begin{tabular}{|c|c|c|c|c|c|}
\hline Section No. & $\begin{array}{c}\text { Section length, } \\
\mathbf{M}\end{array}$ & $\begin{array}{c}\text { Estimate } \\
\text { consumption, } \\
\mathbf{m}^{\mathbf{3}} \mathbf{h}\end{array}$ & $\boldsymbol{d} \times \boldsymbol{S}, \mathbf{m m}$ & $\begin{array}{c}\text { Specific } \\
\text { pressure drop } \\
\frac{\Delta P}{l}, \mathbf{P a} / \mathbf{m}\end{array}$ & $\begin{array}{c}\text { Differential } \\
\text { pressure across } \\
\text { the section } \Delta p, \\
\mathbf{P a}\end{array}$ \\
\hline 1 & 120 & 720 & $219 \times 6$ & 1.6 & 192 \\
\hline 2 & 120 & 120 & $108 \times 4$ & 1.8 & 216 \\
\hline 3 & 120 & 60 & $89 \times 3$ & 1.8 & 216 \\
\hline & $\sum 360$ & & & & $\sum 624$ \\
\hline
\end{tabular}

The pressure loss on the local resistances is assumed equal to $10 \%$ of the linear losses [12]. Preliminary selection of diameters is carried out according to a constant specific pressure drop, which is equal to

$$
\frac{\Delta P}{l}=\frac{624}{1.1 \cdot 360}=1.515 \mathrm{~Pa} / \mathrm{m}
$$

We calculate the nodal correction pressures $\delta p$ and the optimal pressure drops in the sections at the first correction.

$$
\begin{gathered}
\delta p_{I}^{\prime}=\frac{\sum A_{i} \Delta p_{i}^{-1.21}}{1.21 \sum \frac{A_{i} \Delta p_{i}^{-1.21}}{p_{i}}}=\frac{-3.5}{1.21 \cdot 0.043}=67.3 \\
\delta p_{I I}^{\prime}=\frac{\sum A_{i} \Delta p_{i}^{-1.21}}{1.21 \sum \frac{A_{i} \Delta p_{i}^{-1.21}}{p_{i}}}=\frac{-0.6}{1.21 \cdot 0.024}=-20.7 \\
\delta p_{I}^{\prime \prime}=\frac{\sum \delta p_{a . n}\left(\frac{A_{i} \Delta p_{i}^{-1.21}}{p_{i}}\right)}{\sum \frac{A_{i} \Delta p_{i}^{-1.21}}{p_{i}}}=\frac{-20.7 \cdot 0.013}{0.043}=-6.3 \\
\delta p_{I I}^{\prime \prime}=\frac{\sum \delta p_{a . n}\left(\frac{A_{i} \Delta p_{i}^{-1.21}}{p_{i}}\right)_{\text {s.a.n }}}{\sum \frac{A_{i} \Delta p_{i}^{-1.21}}{p_{i}}}=\frac{-67.3 \cdot 0.013}{0.024}=-36.5 \\
\delta p_{I}=\delta p_{I}^{\prime}-\delta p_{I}^{\prime \prime}=-67.3-6.3=73.6 ; \\
\delta p_{I I}=\delta p_{I I}^{\prime}-\delta p_{I I}^{\prime \prime}=-20.7-36.5=-57.5 .
\end{gathered}
$$

Let's calculate the nodal correction pressures and pressure drops in the sections at the second correction:

$$
\delta p_{I}^{\prime}=\frac{\sum A_{i} \Delta p_{i}^{-1.21}}{1,21 \sum \frac{A_{i} \Delta p_{i}^{-1.21}}{p_{i}}}=\frac{-1.18}{1.21 \cdot 0.032}=-30.5
$$




$$
\begin{gathered}
\delta p_{I I}^{\prime \prime}=\frac{0.15}{1.21 \cdot 0.036}=3.44 \\
\delta p_{I}^{\prime \prime}=\frac{\sum \delta p_{a . n}\left(\frac{A_{i} \Delta p_{i}^{-1.21}}{p_{i}}\right)_{\text {s.a.n }}}{\sum \frac{A_{i} \Delta p_{i}^{-1.21}}{p_{i}}}=\frac{-3.4 \cdot 0.016}{0.032}=0.5 ; \\
\delta p_{I I}^{\prime \prime}=\frac{-30.5 \cdot 0.016}{0.036}=-13.5 ; \\
\delta p_{I}=\delta p_{I}^{\prime}+\delta p_{I}^{\prime \prime}=-30.5+0.5=-30 \\
\delta p_{I I}=3.4-13.6=-10.2 .
\end{gathered}
$$

The additional pressure to the drop in the section $\delta p_{s}$ in the absence of an adjacent node is equal to $\delta p$, and in the presence of an adjacent node it is equal to the sum of $\delta p$ and $\delta p$ of an adjacent node with the opposite sign.

Thus:

\begin{tabular}{|c|c|c|c|c|c|c|c|c|}
\hline Node & Section & $\begin{array}{c}\text { Adjacent } \\
\text { nodes }\end{array}$ & $\delta p$ & $\Delta p_{s 2}$ & $\Delta p$ & $\Delta p^{-1.21}$ & $A \Delta p^{-1.21}$ & $\frac{A \Delta p^{1.21}}{\Delta p}$ \\
\hline \multirow{3}{*}{ I } & 1 & & \multirow{2}{*}{-73.6} & -73.6 & -265.6 & $0.12 \cdot 10^{-2}$ & -4.3 & 0.016 \\
\hline & 2 & II & & -16.4 & 199.6 & $0.17 \cdot 10^{-2}$ & 3.12 & 0.016 \\
\hline & & & & \multicolumn{5}{|c|}{ Error $\frac{1.18 \cdot 100}{0.5 \cdot 7.42}=31.8 \%$} \\
\hline \multirow{3}{*}{ II } & 2 & $\mathrm{I}$ & \multirow{3}{*}{-57.2} & 16.4 & 199.6 & $0.17 \cdot 10^{-2}$ & -3.12 & 0.016 \\
\hline & 3 & & & 57.1 & 158.8 & $0.23 \cdot 10^{-2}$ & 3.27 & 0.02 \\
\hline & & & & \multicolumn{5}{|c|}{ Error $\frac{0.15 \cdot 100}{0.5 \cdot 6.39}=4.7 \%$} \\
\hline
\end{tabular}

$$
\begin{gathered}
\Delta p_{s 1}=-30 \\
\Delta p_{s 2}=-30+10.2=-19.8 .
\end{gathered}
$$

Table 2. Determination of gas pressure drops in sections.

\begin{tabular}{|c|c|c|c|c|c|c|c|c|c|}
\hline Node & Section & $\begin{array}{c}\text { Adjacent } \\
\text { nodes }\end{array}$ & $\Delta p$ & $Q^{\mathbf{0 . 3 6 8}}$ & $\boldsymbol{l}^{\mathbf{1 . 2 1}}$ & $\Delta p^{-1.21}$ & $\boldsymbol{A}$ & $\begin{array}{c}\boldsymbol{A} \Delta p^{-} \\
\mathbf{1 . 2 1}\end{array}$ & $\frac{A \Delta p^{1.21}}{\Delta p}$ \\
\hline \multirow{2}{*}{$\mathrm{I}$} & 1 & & -192 & 11.4 & 312 & $0.18 \cdot 10^{-2}$ & 3556.8 & -6.4 & 0.03 \\
\cline { 2 - 10 } & 2 & II & 216 & 5.88 & 312 & $0.16 \cdot 10^{-2}$ & 1834.6 & 2,9 & 0,013 \\
\hline & & & & \multicolumn{7}{|c|}{ Error $\frac{3.5 \cdot 100}{0.5 \cdot 9.3}=75.3 \%$} \\
\hline \multirow{2}{*}{ II } & 2 & I & -216 & 5.88 & 312 & $0.16 \cdot 10^{-2}$ & 1834.6 & -2.9 & 0.013 \\
\hline & 3 & & 216 & 4.55 & 312 & $0.16 \cdot 10^{-2}$ & 1420 & 2.3 & 0.011 \\
\hline & & & & \multicolumn{7}{c|}{ Error $\frac{0.6 \cdot 100}{0.5 \cdot 5.2}=23.1 \%$} \\
\hline
\end{tabular}

Table 3. Iteration 1.

According to Tables 2, 3, 4, the difference in section 1 must be increased, while the diameter of the section will decrease, and the difference in section 3 must be reduced, which will lead to an increase in diameters. 
Table 4. Iteration 2.

\begin{tabular}{|c|c|c|c|c|c|c|c|c|}
\hline Node & Section & $\begin{array}{c}\text { Adjacent } \\
\text { nodes }\end{array}$ & $\delta p$ & $\Delta p_{s 2}$ & $\Delta p$ & $\Delta p^{-1.21}$ & $A \Delta p^{-1.21}$ & $\frac{A \Delta p^{1.21}}{\Delta p}$ \\
\hline \multirow{3}{*}{ I } & 1 & & \multirow{2}{*}{-30} & -30 & -295.6 & $0.11 \cdot 10^{-2}$ & -3.9 & 0.013 \\
\hline & 2 & II & & -19.8 & 179.8 & $0.2 \cdot 10^{-2}$ & 3.7 & 0.02 \\
\hline & & & & \multicolumn{5}{|c|}{ Error $\frac{0.2 \cdot 100}{0.5 \cdot 7.6}=5.2 \%$} \\
\hline \multirow{3}{*}{ II } & 2 & $\mathrm{I}$ & \multirow{3}{*}{-10.2} & 19.8 & 179.8 & $0.2 \cdot 10^{-2}$ & -3.7 & 0.02 \\
\hline & 3 & & & -10.2 & 148.6 & $0.2 \cdot 10^{-2}$ & 3.6 & 0.02 \\
\hline & & & & \multicolumn{5}{|c|}{ Error $\frac{0.1 \cdot 100}{0.5 \cdot 7.3}=2.7 \%$} \\
\hline
\end{tabular}

Table 5. Final calculation of diameters.

\begin{tabular}{|c|c|c|c|}
\hline Section No. & $\boldsymbol{d}, \mathbf{\text { мм }}$ & Specific pressure drop $\frac{\Delta P}{l}, \mathbf{P a} / \mathbf{m}$ & $\begin{array}{c}\text { Differential pressure } \\
\text { across the section } \Delta p, \mathbf{P a}\end{array}$ \\
\hline 1 & $194 \times 6$ & 2.1 & 252 \\
\hline 2 & $108 \times 4$ & 1.9 & 228 \\
\hline 3 & $90 \times 4$ & 1.2 & 144 \\
\hline \multicolumn{2}{|r|}{} & $\sum 624$ \\
\hline
\end{tabular}

Let's calculate the comparison of costs by $\frac{\Delta P}{l}=$ const:

$$
k=b \sum d l=b(21.9 \cdot 120+10.8 \cdot 120+8.9 \cdot 120)=b \cdot 4992 .
$$

Economics:

$$
k=b(1.94 \cdot 120+10.8 \cdot 120+9 \cdot 120)=b \cdot 4704 .
$$

The cost reduction is about $5.77 \%$.

Let's calculate comparisons of metal inputs by $\frac{\Delta P}{l}=$ const:

$$
M=\sum d \delta l=(21.9 \cdot 0.6 \cdot 120+10.8 \cdot 0.4 \cdot 120+8.9 \cdot 0.3 \cdot 120) \cdot c=2415.6 .
$$

Economics:

$$
M=(19.4 \cdot 0.6 \cdot 120+10.8 \cdot 0.4 \cdot 120+9 \cdot 0.4 \cdot 120) \cdot c=2347.2
$$

Metal savings are about $2.8 \%$.

\section{Conclusions}

As a result of the calculations, the pressure losses are distributed between successive sections and the cost of the gas distribution system reduces by $2.8 \%$. In this case, the gas pressure drop in section 1 increased, which means that the diameter value decreased from $219 \times 6$ to $194 \times 6$. In section 3 , the gas pressure drop decreased and this led to an increase in diameter from $89 \times 4$ to $90 \times 4$.

\section{References}

1. A. V. Zhila, E. Solovyeva, Business Technologies for Sustainable Urban Development, 3-16 (2018) 
2. E. Solovyeva, E3S Web of Conferences 91, 07016 (2019)

3. W. Yu, K. Wen, J. Gong, Y. Li, W. Huang, Proceedings of the Biennial International Pipeline Conference (2018)

4. O. Gnezhdilova, A. Malysheva, A. Gagulina, A. Ovsienko, E3S Web Conf. XXII International Scientific Conference 97 (2019)

5. S.A. Gerelov, Complex system of construction of gas distribution pipelines from polymer materials (Moscow, 2002)

6. On measures to ensure safety when using and maintaining indoor and in-apartment gas equipment. Resolution of the Government of the Russian Federation of May 14, No. 410 (2013) http://www.consultant.ru

7. F. Chen, C. Wu, Frontiers in Energy 2, 213-223 (2020)

8. On approval of technical regulations on the safety of gas distribution and gas consumption networks. Decree of the Government of the Russian Federation of October 29, No. 870 (2010) http://www.consultant.ru

9. O.N. Medvedeva, A.Y. Chilikin, IOP Conference Series: Materials Science and Engineering 042049 (2018)

10. V. Zhila, FarEastCon 8934059 (2019)

11. A.A. Malysheva, E3S Web of Conferences 135 (2019) 